\title{
Data-based PID control of flexible joint robot using adaptive safe experimentation dynamics algorithm
}

\author{
Mohd Ashraf Ahmad, Haszuraidah Ishak, Ahmad Nor Kasruddin Nasir, Normaniha Abd Ghani \\ Faculty of Electrical and Electronics Engineering Technology, University Malaysia Pahang, Malaysia
}

\begin{tabular}{l} 
Article Info \\
\hline Article history: \\
Received Dec 30, 2019 \\
Revised Mar 8, 2020 \\
Accepted Jun 23, 2020 \\
\hline
\end{tabular}

Keywords:

Data-based control

Flexible mechanism

Model-free method

PID controller

Vibration reduction

\begin{abstract}
This paper proposes the data-based PID controller of flexible joint robot based on adaptive safe experimentation dynamics (ASED) algorithm. The ASED algorithm is an enhanced version of SED algorithm where the updated tuning variable is modified to adapt to the change of the objective function. By adopting the adaptive term to the updated equation of SED, it is expected that the convergence accuracy can be further improved. The effectiveness of the ASED algorithm is verified to tune the PID controller of flexible joint robot. In this flexible joint control problem, two PID controllers are utilized to control both rotary angle tracking and vibration of flexible joint robot. The performance of the proposed data-based PID controller is assessed in terms of trajectory tracking of angular motion, vibration reduction and statistical analysis of the pre-defined control objective function. The simulation results showed that the data-based PID controller based on ASED is able to produce better control accuracy than the conventional SED based method.
\end{abstract}

This is an open access article under the CC BY-SA license.

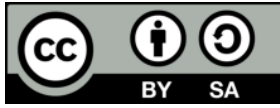

Corresponding Author:

Mohd Ashraf Ahmad,

Faculty of Electrical and Electronics Engineering Technology,

University Malaysia Pahang,

26600, Pekan, Pahang, Malaysia.

Email: mashraf@ump.edu.my

\section{INTRODUCTION}

Data-based control of flexible joint robot have received great attentions by many researchers as compared to model-based control schemes $[1,2]$. This is due to huge effort is required to obtain an accurate model of such complex system [3] and further design the model-based controller. Meanwhile, data-based control of flexible joint robot can be divided into two classes, which are feedforward data-driven control and feedback data-driven control. The examples of feedforward data-based control are input shaping and filtering techniques [4-7]. Here, the techniques utilize the information of vibration frequencies to design the input shaper and filter after generating a bang-bang input torque to the flexible robot system. Meanwhile, in the feedback data-based control, many researchers apply the multi-agent-based optimization tools to tune the feedback control, i.e., PID, based on the input and output data [8-11]. The advantage of feedforward data-based control is that it only requires one shot of output data to design the input shaping or filtering control schemes as compared to feedback data-driven control, which requires large number of data set. However, the feedforward data-based control is not able to handle any disturbances, while the feedback data-based control scheme can successfully handle any disturbances due to its closed-loop structure.

Recently, in the feedback data-based control schemes, there are various of optimization tools used to tune a pre-defined control structure. For example, particle swarm optimization (PSO) has been widely applied to tune various controller for flexible robot. In [12], they used PSO to tune the dynamic neural network for two-link flexible robot robot. In particular, an improved PSO/Bayesian regularization (BR) has 
been proposed with the integration of extremum response surface method and artificial neural network. In [13], new gains tuning and impedance control method have been applied for flexible base moving robots. Here, a new online PSO for gain tuning of impedance control at the contact moments of end effector has been proposed. In [14], they applied PSO to tune the linear and nonlinear active rejection controller. Meanwhile, genetic algorithm (GA) has been used to tune the PID with input shaping controller for input tracking and vibration control of flexible robot [15]. Several similar works on applying GA for tuning controller of flexible robot can be found in [16-18]. Furthermore, evolutionary algorithm (EA) has been used for system identification of flexible robot and controller tuning [19]. Similarly, evolutionary computation (EC) has also been applied for tip position controller of two-link flexible robot [20]. Meanwhile, bees algorithm and artificial bee colony have been used to tune the hierarchical PID [21] and intelligent PID [22] of single-link flexible robot, respectively.

Based on the above literature, the main ineluctable limitation in their works is most of the methods requires heavy computation time to obtain the optimal controller parameter. This is because, in the multi-agent-based optimization, the computation times per iteration are proportional to the number of agents. Hence, it motivates us to propose a tuning strategy that requires less computation time, such as single agent-based optimization tools. So far, there are various single agent-based optimization tools that have been applied for data-based control such as simultaneous perturbation stochastic approximation (SPSA) [23], simulated annealing (SA) [24], random search (RS) [25] and safe experimentation dynamics (SED) [26, 27]. Based on findings in [28], it is shown that the adaptive safe experimentation dynamics (ASED) is the most significant single-agent based optimization tool as compared to the other single-agent-based optimization tools due to its simplicity, memory based structure and less number of coefficients. In addition, there are still no reported works adopt the ASED based method for controlling the flexible joint robot. Thence, it is worth to see the effectiveness of the ASED for data-based control of flexible joint robot.

This paper presents the data-based PID controller of flexible joint robot using ASED algorithm. In the new version of SED, the updated tuning variable is enhanced to adapt with the variation of the objective function during the tuning process [28]. The effectiveness of the ASED algorithm is verified for finding the optimal PID controller of flexible joint robot. In particular, two PID controllers are utilized to control both angular tracking and vibration of elastic joint robot. The performance of the proposed data-based PID controller is assessed in terms of trajectory tracking of angular motion, vibration reduction and statistical analysis of the pre-defined control objective function. The results of the simulation showed that the databased PID controller based on ASED algorithm could exhibit slighltly improvement in the control accuracy than the standard SED algorithm.

\section{RESEARCH METHOD}

The synthesize of data-based PID controller of flexible joint robot using ASED is explained in this section. Firstly, the problem setting of data-based PID controller of flexible joint robot is presented. Here, the structure of conventional PID controller and its parameter are explained. Secondly, we show the procedure to find the optimal PID parameters using ASED such that the given control objective function is minimized.

\subsection{Problem setting of data-based PID controller}

Figure 1 shows block diagram of the PID control system for flexible joint robot. In the Figure 1, the symbols $G, Y_{R}(t), Y(t), V(t)$, and $\theta(t)$ are defined as flexible joint robot plant, reference, rotary angle, control input and oscillation angle, respectively. Note that the flexible joint robot is a class of underactuated system since only one control input is used to regulate both rotary angle and oscillation angle.

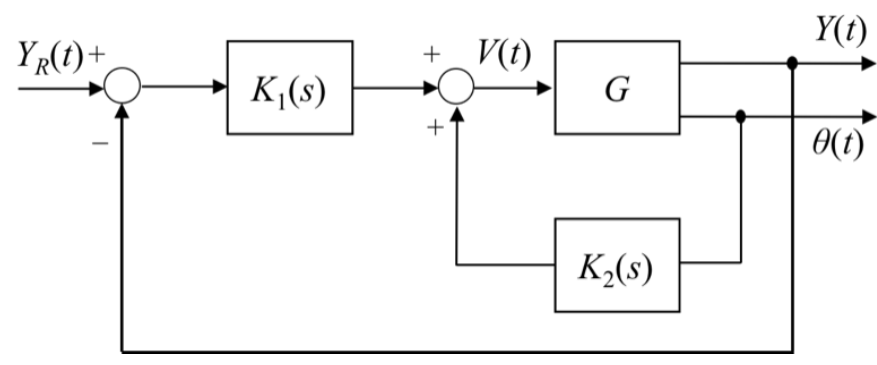

Figure 1. Control system block diagram of flexible joint robot 
Furthermore, two PID controllers, which are denoted by the symbols $K_{1}(s)$ and $K_{2}(s)$ are fed back from the rotary angle and the oscillation angle, respectively. The detailed expression of both PID controllers are given by (1) and (2).

$$
\begin{aligned}
& K_{1}(s)=P_{1}\left(1+\frac{1}{I_{1} s}+\frac{D_{1} s}{1+\left(D_{1} / N_{1}\right) s}\right) \\
& K_{2}(s)=P_{2}\left(1+\frac{1}{I_{2} s}+\frac{D_{2} s}{1+\left(D_{2} / N_{2}\right) s}\right)
\end{aligned}
$$

where $P_{1}, P_{2} \in \mathbf{R}, I_{1}, I_{2} \in \mathbf{R}, D_{1}, D_{2} \in \mathbf{R}$ and $N_{1}, N_{2} \in \mathbf{R}$ are the proportional gains, integral gains, derivative gains and filter coefficients, respectively. Our aim is to design data-based PID controller such that the rotary angle follow the desired reference trajectory with minimum oscillation angle. Therefore, the control objective function is designed as (3)

$$
J(\boldsymbol{P}, \boldsymbol{I}, \boldsymbol{D}, \boldsymbol{N})=w_{1} \hat{E}+w_{2} \hat{\theta}+w_{3} \widehat{V}
$$

where:

$$
\begin{aligned}
& \hat{E}=\int_{t_{0}}^{t_{f}}\left|Y_{R}(t)-Y(t)\right|^{2} d t, \\
& \hat{\theta}=\int_{t_{0}}^{t_{f}}|\theta(t)|^{2} d t, \\
& \hat{V}=\int_{t_{0}}^{t_{f}}|V(t)|^{2} d t,
\end{aligned}
$$

for the time duration of $\left[t_{0}, t_{f}\right]$. In (3), the symbols $\boldsymbol{P}=\left[\begin{array}{ll}P_{1} P_{2}\end{array}\right]^{\mathrm{T}}, \boldsymbol{I}=\left[\begin{array}{ll}I_{1} & I_{2}\end{array}\right]^{\mathrm{T}}, \boldsymbol{D}=\left[\begin{array}{ll}D_{1} & D_{2}\end{array}\right]^{\mathrm{T}}$, and $\boldsymbol{N}=\left[N_{1} N_{2}\right]^{\mathrm{T}}$, which contribute to 8 tuning variables in PID controllers. In order to properly regulate this kind of multi-objective function in (3), several weight coefficients are introduced, which is denoted by the symbols $w_{1} \in \mathbf{R}, w_{2} \in \mathbf{R}$ and $w_{3} \in \mathbf{R}$. Finally, the problem setting for data-based PID controller is given by:

Problem 1. Based on the given plant $G$ with PID controller structure in Figure 1 and the available input and output data, find the PID controllers $K_{1}(s)$ and $K_{2}(s)$ such that the objective function in (3) is minimized.

\subsection{Data-based PID utilizing adaptive safe experimentation dynamics}

In this section, the proposed ASED algorithm is used for finding the optimal PID controller of flexible joint control problem. Initially, the conventional SED algorithm that was firstly introduced by Marden et. al., [29] is reviewed. Next, the enhanced version of SED, which is called ASED is presented. Ultimately, the procedure to utilize the ASED algorithm in finding the optimal PID controller of flexible joint robot is shown. Since our aim is to minimize the objective function in (3), we consider the minimization optimization problem as (7).

$$
\min _{z \in \mathbf{R}^{n}} L(\mathbf{z})
$$

where $L: \mathbf{R}^{n} \rightarrow \mathbf{R}$ is an unknown loss function with the tuning variable vector $\boldsymbol{z} \in \mathbf{R}^{n}$. The SED algorithm [29] updates $\boldsymbol{z} \in \mathbf{R}^{n}$ to find an optimal solution $z^{* \in \mathbf{R}^{n}}$ of (7). The updated equation of SED algorithm is expressed by (8).

$$
z_{i}(k+1)= \begin{cases}h\left(\bar{z}_{i}-K_{g} r_{1}\right) & \text { if } r_{1} \leq E, \\ \bar{z}_{i} & \text { if } r_{1}>E,\end{cases}
$$

where $k=0,1, \ldots$, is iteration number, $z_{i} \in \mathbf{R}$ is the $i$ th element of $\mathbf{z} \in \mathbf{R}^{n}$, and $\bar{z}_{i} \in \mathbf{R}$ is the $i$ th iteration of $\overline{\mathbf{z}} \in \mathbf{R}^{n}$. The symbol $\overline{\boldsymbol{z}}$ is denoted as the current best value of the tuning variable. The symbol $K_{g}$ is the size of interval to determine the random steps on $z_{i} \in \mathbf{R}$, the symbol $E$ is the probability to change the element of the tuning variable and $r_{1} \in \mathbf{R}$ is the random value that is generated uniformly between 0 and 1 . In (8), the function $h$ is given by (9). 


$$
h(.)=\left\{\begin{array}{c}
Z_{\text {max }}, \bar{z}_{i}-K_{g} r_{2}>Z_{\text {max }} \\
\bar{z}_{i}-K_{g} r_{2}, \quad Z_{\min } \leq \bar{Z}_{i}-K_{g} r_{2} \leq Z_{\text {max }} \\
Z_{\text {min }}, \bar{z}_{i}-K_{g} r_{2}<Z_{\text {min }}
\end{array}\right.
$$

where the pre-determined minimum and maximum of tuning variable are denoted by $Z_{\min }$ and $Z_{\text {max }}$, respectively, and $r_{2}$ is another random number that is generated independently from $r_{1}$. The step-by-step procedure to execute the SED algorithm are given by:

S1: Determine the values of $Z_{\text {min }}, Z_{\text {max }}, K_{g}$ and $E$. Set the initial conditions of the tuning variable $\boldsymbol{z}(0)$. Calculate the loss function $L(\mathbf{z}(0))$ and set $\overline{\boldsymbol{z}}=\mathbf{z}(0)$ and $\bar{L}=L(\mathbf{z}(0))$.

S2: Execute $\overline{\boldsymbol{z}}=\boldsymbol{z}(k)$ and $\bar{L}=L(\mathbf{z}(k))$ when the value $L(\boldsymbol{z}(k))<\bar{L}$. Otherwise, go to $\mathbf{S 3}$.

S3: Generate random numbers $r_{1}$ and $r_{2}$ independently and execute the updated equation in (8).

S4: Calculate the loss function $L\left(z_{i}(k+1)\right)$.

S5: If the pre-defined termination criterion (such as based on the designated maximum number of iteration $\left.k_{\max }\right)$ is satisfied, the algorithm terminates with the optimum tuning variable $\mathbf{z}^{*}:=$ $\arg \min _{z \in\{z(0), z(1), \ldots, z(k+1)\}} L(\mathbf{z})$. Otherwise, repeat $\mathbf{S 2}$.

Based on our preliminary study in the controller tuning process, there is a probability that the obtained control tuning parameter converges too early which may degrade the overall control performance accuracy. It clarifies that the conventional SED algorithm is still not enough to achieve good convergence accuracy. This is because the updated tuning variable in (8) is only depends on $K_{g}$ and uniformly distributed numbers $r_{1}$ and $r_{2}$, which make it similar to basic random search strategy. Therefore, in order to solve the above issue, we adopt the ASED from [28] as the optimization tool for data-based PID controller of flexible joint robot. In particular, the updated in (8) is modified as (10).

$$
z_{i}(k+1)= \begin{cases}h\left(\bar{z}_{i}-K_{g} r_{1}\right)+K_{a}\left(\frac{L\left(z_{i}(k)\right)-\bar{L}}{L\left(z_{i}(k)\right)}\right) & \text { if } r_{1} \leq E \\ \bar{z}_{i}+K_{a}\left(\frac{L\left(z_{i}(k)\right)-\bar{L}}{L\left(z_{i}(k)\right)}\right) & \text { if } r_{1}>E\end{cases}
$$

where $K_{a}$ is another gain to adjust the adaptive mechanism. Note that, by using the new updated in (10), the probability of perturb each element of tuning variable is increased, which could lead to extra effort of finding the optimal tuning variable [28]. Furthermore, by using the new updated in (10), the procedure to implement ASED for data-based PID controller of flexible joint robot is given as follow

Step 1: Determine the maximum iteration $k_{\max }$ and consider $L(\mathbf{z})=J(\boldsymbol{P}, \boldsymbol{I}, \boldsymbol{D}, \boldsymbol{N})$ and $z_{i}=\log \psi_{i}$. Note that $\psi=\left[\begin{array}{llllllll}P_{1} & I_{1} & D_{1} & N_{1} & P_{2} & I_{2} & D_{2} & N_{2}\end{array}\right]^{\mathrm{T}}$ is the tuning variable vector and $\psi_{i}=10^{z_{i}}(i=1,2, \ldots, 8)$.

Step 2: Execute the ASED algorithm.

Step 3: After $k_{\max }$ is achieved, obtain the optimal tuning variable $\mathbf{z}^{*}=\bar{z}$. Then, implement to PID controller $\psi^{*}=\left[\begin{array}{llll}10^{\bar{z}_{1}} & 10^{\bar{z}_{2}} & \cdots & 10^{\bar{z}_{8}}\end{array}\right]^{\mathrm{T}}$ in Figure 1.

\section{RESULTS AND ANALYSIS}

The efficacy of the ASED algorithm in finding the optimal PID controller flexible joint robot is demonstrated in this section. In particular, the convergence curve response of the objective function in (3), the rotary angle, oscillation angle and input responses, and the statistical analysis of objective function, integral square error and integral square input are presented and analyzed. In this study, 25 trials are considered to evaluate the statistical performances of ASED. In this study, the model of the flexible joint plant $G$ is taken from [30]. The reference $Y_{R}(t)$ is expressed by (11).

$$
Y_{R}(t)=25(\tanh (0.005(t-0.7))+1)
$$

for $0<t<4$. Here, the objective is to find the optimal PID controller such that the rotary angle can follow the pre-defined trajectory in (11) with minimum oscillation angle. The coefficients of SED are given as $K_{g}=0.01, E=0.65, Z_{\min }=-3, Z_{\max }=3$. Meanwhile, the coefficients of ASED are same with SED, except for $K_{a}=0.03$. The weighting coefficients are set as $w_{1}=400, w_{2}=200$ and $w_{3}=2$. The initial values of the tuning variable is given as $z(0)=\left[\begin{array}{llllllll}0.5 & 1.0 & 0.0 & 1.0 & 0.0 & 1.0 & 0.0 & 2.0\end{array}\right]^{\mathrm{T}}$. Note that the initial values are selected after performing several preliminary experiments by guaranteeing a stable response is obtained.

Figure 2 shows the response of the objective function convergence after 300 iterations to produce

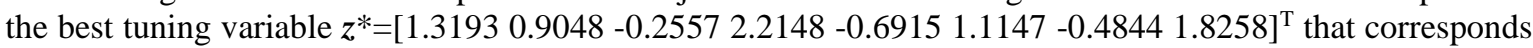


to $\psi^{*}=\left[\begin{array}{llllllll}20.8589 & 8.0320 & 0.5551 & 163.9941 & 0.2035 & 13.0240 & 0.3278 & 66.9505\end{array}\right]^{\mathrm{T}}$. Note that the convergence response in Figure 2 is the best convergence response out of 25 trials. It shows that the ASED based method is able to minimize the objective function in (3) and produce better output and input responses, which can be clearly seen in Figures 3, 4 and 5. Here, the red-dotted line corresponds to the response of the controller based on initial PID parameters $(k=0)$, while the straight black line refers to optimal PID parameters $(k=300)$. In Figure 5, it shows that the data-based PID utilizing ASED successfully improves the rotary angle tracking, with very minimal overshoot and almost zero steady state error. In terms of oscillation angle as shown in Figure 4, the optimal PID controller can minimize the oscillation angle faster than the initial PID controller, which is within 3 seconds. Furthermore, it produces slightly lower magnitude of oscillation angle, which is from -0.9 degree to 3.6 degree, as compared to initial PID controller. Similarly, the control input of optimal PID controllers produces lower settling time but with higher magnitude of input as compared to the initial PID parameter.

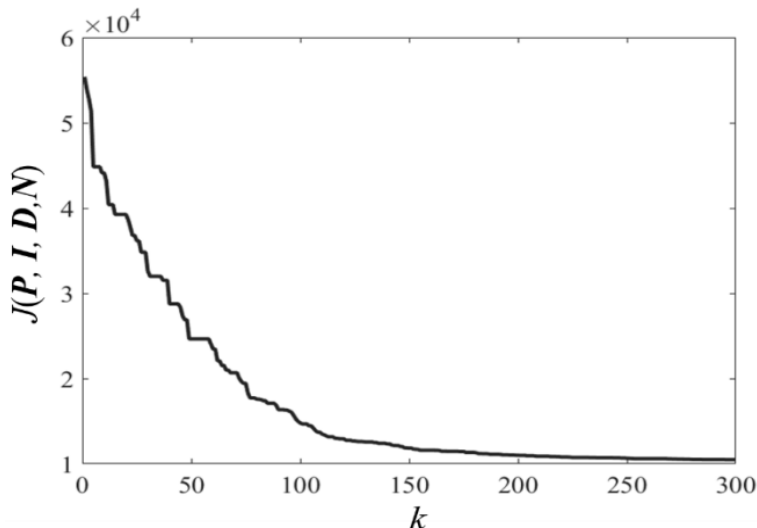

Figure 2. Convergence curve response

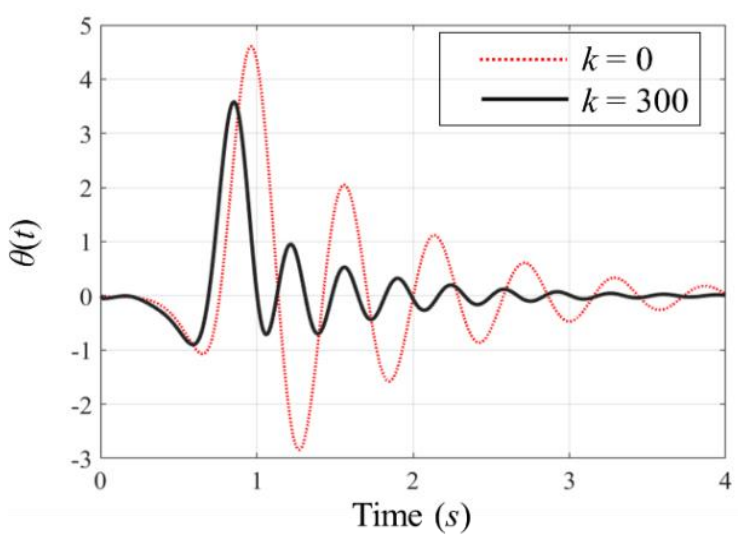

Figure 4. Oscillation angle responses

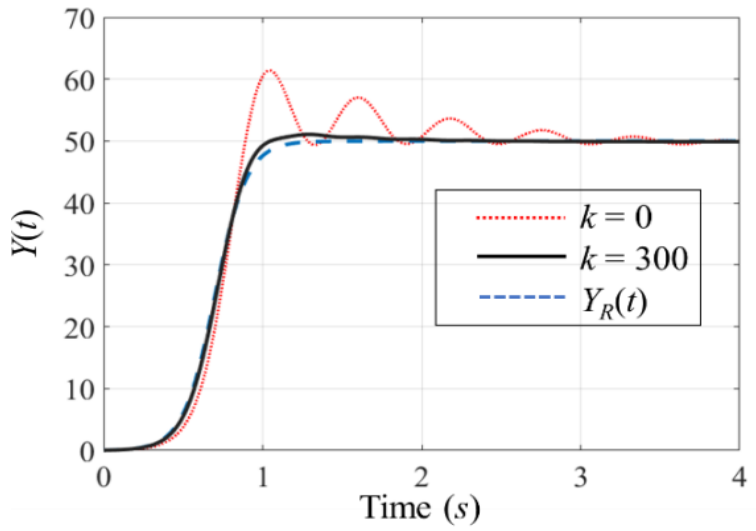

Figure 3. Rotary angle responses

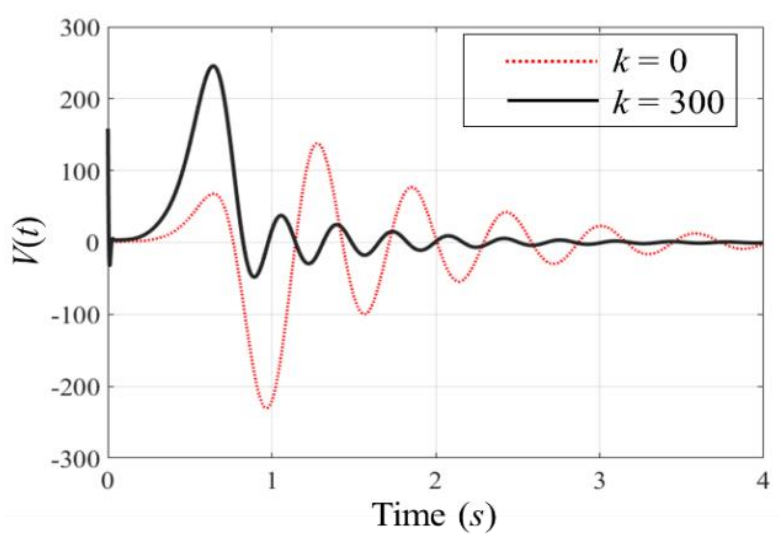

Figure 5. Input responses

Furthermore, the control performances of the data-based PID based on ASED are also compared with the data-based PID based on standard SED, in terms of the statistical analysis of the objective function, integral square error and integral square input. Table 1 depicts the performance comparison for both methods after 25 trials. Here, the numerical values with bold in Table 1 indicate the best performance. It shows that the ASED produce slightly better statistical values (in terms of mean, best, worst and Std.) in the objective function and tracking error than the standard SED. Moreover, the ASED also yields slightly lower mean and best values in the control input analysis than the standard SED. Therefore, it justifies that the proposed ASED can yield improvement in the PID control accuracy, especially on the objective function and the tracking error than the standard SED. 
Table 1. Statistical performances between standard SED and ASED

\begin{tabular}{cccc}
\hline \multicolumn{2}{c}{ Algorithm } & Standard SED & ASED \\
\hline & Mean & 10.6071 & $\mathbf{1 0 . 5 9 9 0}$ \\
$J(\boldsymbol{P}, \boldsymbol{I}, \boldsymbol{D}, \boldsymbol{N})\left(\times 10^{3}\right)$ & Best & 10.4915 & $\mathbf{1 0 . 4 8 7 9}$ \\
& Worst & 10.8443 & $\mathbf{1 0 . 8 0 4 9}$ \\
& Std. & 0.0788 & $\mathbf{0 . 0 7 4 8}$ \\
& Mean & 3.3807 & $\mathbf{3 . 3 7 1 2}$ \\
$\hat{E}+\hat{\theta}$ & Best & 3.2280 & $\mathbf{3 . 2 1 9 1}$ \\
& Worst & 3.7808 & $\mathbf{3 . 7 4 9 0}$ \\
& Std. & 0.1312 & $\mathbf{0 . 1 1 5 9}$ \\
$\hat{V}\left(\times 10^{3}\right)$ & Mean & 4.8453 & $\mathbf{4 . 8 4 2 9}$ \\
& Best & 4.7898 & $\mathbf{4 . 7 8 2 6}$ \\
& Worst & $\mathbf{4 . 9 0 7 8}$ & 4.9203 \\
& Std. & $\mathbf{0 . 0 3 5 2}$ & 0.0355 \\
\hline
\end{tabular}

\section{CONCLUSION}

In this study, a data-based PID utilizing ASED algorithm for flexible joint robot has been presented. The results demonstrated that the proposed ASED based method has a good capability in the improvement of the PID control accuracy. In particular, it is shown that the proposed ASED based method is able to produce lower values of objective function, integral square error and integral square input as compared to standard SED based method. The findings also be justified through the responses of the rotary angle tracking and the oscillation angle. In the future, the ASED algorithm can be extended to tune various types controller such as fuzzy logic controller and neural network controller.

\section{ACKNOWLEDGEMENTS}

The authors gratefully acknowledged Universiti Malaysia Pahang, especially Research and Innovation Department under UMP internal grant RDU190325 for giving the financial support.

\section{REFERENCES}

[1] M. Khairudin, Z. Mohamed, A. R. Husain, "Dynamic model and robust control of flexible link robot manipulator," TELKOMNIKA Telecommunication, Computing, Electronics and Control, vol. 9, no. 2, pp. 279-286, Aug 2011.

[2] M. Khairudin, Z. Mohamed, A.R. Husain, "System identification and LMI based robust PID control of a two-link flexible manipulator," TELKOMNIKA Telecommunication, Computing, Electronics and Control, vol. 12, no. 4, pp. 829-838, Dec 2014.

[3] M. Khairudin, Z. Mohamed, A. R. Husain, M. A. Ahmad, "Dynamic modelling and characterisation of a two-link flexible robot manipulator," Journal of low frequency noise, vibration and active control, vo. 29, no. 3, pp. 207-219, Sep 2010.

[4] Z. Mohamed, M. A. Ahmad, "Hybrid input shaping and feedback control schemes of a flexible robot manipulator," IFAC Proceedings Volumes, vol. 41, no. 2, pp. 11714-11719, 2008.

[5] W. Chatlatanagulchai, D. Kijdech, T. Benjalersyarnon, S. Damyot, "Quantitative feedback input shaping for flexible-joint robot manipulator," Journal of Dynamic Systems, Measurement, and Control, vol. 138, no. 6, pp. 1-13, 2016.

[6] T. Solatges, S. Rubrecht, M. Rognant and P. Bidaud, "Adaptive input shaper design for flexible robot manipulators," 2017 IEEE/RSJ International Conference on Intelligent Robots and Systems (IROS), Vancouver, BC, pp. 444-449, 2017.

[7] H. Ghorbani, K. Alipour, B. Tarvirdizadeh, A. Hadi, "Comparison of various input shaping methods in rest-to-rest motion of the end-effecter of a rigid-flexible robotic system with large deformations capability," Mechanical Systems and Signal Processing, vol. 118, pp. 584-602, March 2019.

[8] B. Subudhi, A. S. Morris, "Soft computing methods applied to the control of a flexible robot manipulator," Applied Soft Computing, vol. 9, no. 1, pp. 149-158, Jan 2009.

[9] H. A. Elkaranshway, E. H. Bayoumi, H. M. Soliman, "PSO-based robust PID control for flexible manipulator systems," International Journal of Modelling, Identification and Control, vol. 14, no. 1-2, pp. 1-12, Sep 2011.

[10] J. Annisa, I. Z. M. Darus, M. O. Tokhi and S. Mohamaddan, "Implementation of PID Based Controller Tuned by Evolutionary Algorithm for Double Link Flexible Robotic Manipulator," 2018 International Conference on Computational Approach in Smart Systems Design and Applications (ICASSDA), Kuching, pp. 1-5, 2018.

[11] A. Lismonde, V. Sonneville, O. Brüls, "A geometric optimization method for the trajectory planning of flexible manipulators," Multibody System Dynamics, vol. 47, no. 4, pp. 347-362, 2019.

[12] L. K. Song, C. W. Fei, G. C. Bai, L. C. Yu, "Dynamic neural network method-based improved PSO and BR algorithms for transient probabilistic analysis of flexible mechanism," Advanced Engineering Informatics, vol. 33, pp. 144-153, 2017. 
[13] M. Salehi, G. R. Vossoughi, M. Vajedi and M. Brooshaki, "Impedance control and gain tuning of Flexible Base Moving Manipulators using PSO method," 2008 International Conference on Information and Automation, Changsha, pp. 458-463, 2008.

[14] A. J. Humaidi, H. M. Badir, "Linear and nonlinear active rejection controllers for single-link flexible joint robot manipulator based on PSO tuner," ARPN Journal of Engineering and Applied Sciences, vol. 13, no. 6, pp. 2272-2278, 2018.

[15] F. M. Aldebrez, M. S. Alam and M. O. Tokhi, "Input-Shaping with GA-Tuned PID for Target Tracking and Vibration Reduction," Proceedings of the 2005 IEEE International Symposium on, Mediterrean Conference on Control and Automation Intelligent Control, 2005., Limassol, pp. 485-490, 2005.

[16] B. A. M. Zain, M. O. Tokhi and S. F. Toha, "PID-Based Control of a Single-Link Flexible Manipulator in Vertical Motion with Genetic Optimisation," 2009 Third UKSim European Symposium on Computer Modeling and Simulation, Athens, pp. 355-360, 2009.

[17] B. A. M. Zain, M. O. Tokhi, "Genetic algorithm optimization of pid controller for a flexible manipulator," In Advances In Mobile Robotics pp. 431-438, 2008.

[18] M. N. H. Siddique and M. O. Tokhi, "GA-based neuro-fuzzy controller for flexible-link manipulator," Proceedings of the International Conference on Control Applications, Glasgow, UK, vol. 1, pp. 471-476, 2002.

[19] M. S. Saad, H. Jamaluddin, I. Z. M. Darus, "Active vibration control of a flexible beam using system identification and controller tuning by evolutionary algorithm," Journal of Vibration and Control, vol. 21, no. 10, pp. 2027-2042, 2015.

[20] B. Subudhi, S. Ranasingh, A. Swain, "Evolutionary computation approaches to tip position controller design for a two-link flexible manipulator," Archives of Control Sciences, vol. 21, no. 3, pp. 269-285, 2011.

[21] D. T. Pham, E. Koç, M. Kalyoncu, M. Tınkır, "Hierarchical PID controller design for a flexible link robot manipulator using the bees algorithm," Proceedings of 6th International Symposium on Intelligent Manufacturing Systems, pp. 1-6, 2008.

[22] M. S. Hadi, I. Z. M. Darus, M. O. Tokhi, M. F. Jamid, "Active vibration control of a horizontal flexible plate structure using intelligent proportional-integral-derivative controller tuned by fuzzy logic and artificial bee colony algorithm," Journal of Low Frequency Noise, Vibration and Active Control, pp. 1-13, 2019.

[23] M. A. Ahmad, S. I. Azuma, T. Sugie, "Performance analysis of model-free PID tuning of MIMO systems based on simultaneous perturbation stochastic approximation," Expert Systems with Applications, vol. 41, no. 14, pp. 6361-6370, Oct 2014.

[24] L. F. Fraga-Gonzalez, R. Q. Fuentes-Aguilar, A. Garcia-Gonzalez, G. Sanchez-Ante, "Adaptive simulated annealing for tuning PID controllers," AI Communications, vol. 30, no. 5, pp. 347-362, 2017.

[25] L. Nuñez, R. G. Regis, K. Varela, "Accelerated random search for constrained global optimization assisted by radial basis function surrogates," Journal of Computational and Applied Mathematics, vol. 340, pp. 276-295, Oct 2018.

[26] J. R. Marden, S. D. Ruben and L. Y. Pao, "A Model-Free Approach to Wind Farm Control Using Game Theoretic Methods," in IEEE Transactions on Control Systems Technology, vol. 21, no. 4, pp. 1207-1214, July 2013.

[27] M. I. F. Mohd Hanif, M. H. Suid, M. A. Ahmad, "A Piecewise Affine PI Controller for Buck Converter Generated DC Motor," International Journal of Power Electronics and Drive Systems (IJPEDS), vol. 10, no. 3, pp. 1419-1426, Sep 2019.

[28] M. R. Ghazali, M. A. Ahmad, R. M. T. Raja Ismail, "Adaptive safe experimentation dynamics for data-driven neuroendocrine-PID control of MIMO systems," IETE Journal of Research, in press, pp. 1-14, 2019.

[29] J. R. Marden, H. P. Young, G. Arslan, J. S. Shamma, "Payoff-based dynamics for multiplayer weakly acyclic games," SIAM Journal on Control and Optimization, vol. 48, no. 1, pp. 373-396, 2009.

[30] “Rotary Flexible Joint User Manual," Quanser Inc., Markham, ON, Canada, 2008. 